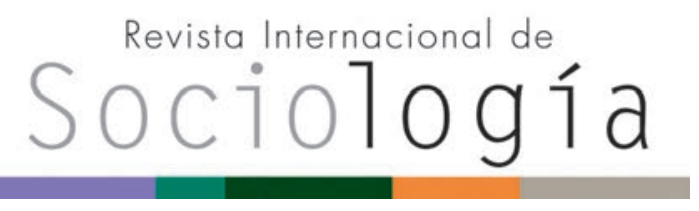

Revista Internacional de Sociología RIS vol. 75 (4), e079, octubre-diciembre, 2017, ISSN-L:0034-9712 doi: http://dx.doi.org/10.3989/ris.2017.75.4.17.05

\section{EXPERIMENTANDO CON LA TIERRA. Geotermia, no-conocimiento y transiciones energéticas como experimentos a la intemperie}

\author{
Matthias Gross \\ Friedrich-Schiller-University Jena, Germany. \\ matthias.gross@ufz.de \\ ORCID iD: http://orcid.org/0000-0002-3716-7512
}

\section{EXPERIMENTING WITH EARTH. Geothermics, non-knowledge and energy transitions as experiments in the wild}

\author{
MANUEL TIRONI \\ Pontificia Universidad Católica de Chile, Chile / Centro de Energía UC, Chile. \\ metironi@uc.cl \\ ORCID iD: http://orcid.org/0000-0003-2733-8071
}

Cómo citar este artículo / Citation: Gross, M. y M. Tironi. 2017. "Experimentando con la tierra. Geotermia, no-conocimiento y transiciones energéticas como experimentos a la intemperie". Revista Internacional de Sociología 75 (4): e079. doi: http://dx.doi.org/10.3989/ris.2017.75.4.17.05

\section{RESUMEN}

Los combustibles fósiles no-renovables hicieron posible lo que se ha llamado el mundo moderno. Dado que los recursos no renovables son cada vez más difíciles y costosos de extraer, la transición a fuentes de energías renovables es una tarea crucial. En orden a enmarcar posibles caminos hacia la innovación responsable y transición dentro de las sociedades democráticas, este artículo se construye en torno al concepto de experimentos "terrenales" o fuera-del-laboratorio para destacar algunas de las condiciones y procesos que pueden promover procesos colectivos de aprendizaje. Esto es hecho por medio de ejemplos empíricos con respecto al establecimiento de sistemas de energía geotérmicas. El artículo trabajará sobre la noción sociológica de experimentos terrenales, entendiéndolos como parte sustantiva de la sociedad, con el fin de destacar críticamente qué podría significar una "ciencia" así concebida para una ciudadanía democráticamente constituida. El artículo mostrará cómo estos procesos experimentales lidian con cuestiones referentes a la aceptabilidad y la gobernanza democrática, y de qué manera aparecen y se gestionan los límites de la experimentalidad de cara a sus desafíos políticos. Mostramos que los "experimentos a la intemperie" tendrán que enfrentarse con incertidumbres crecientes y la inevitable ignorancia que complica las condiciones para la transición hacia energías renovables, pero que también pueden abrir nuevos caminos para involucrar a la ciudadanía en procesos de exploración tecnocientífica.
\end{abstract}

\section{Palabras Clave}

Energías renovables; Experimentación; Participación; Sustentabilidad.
Copyright: (C) 2017 CSIC. Este es un artículo de acceso abierto distribuido bajo los términos de la licencia Creative Commons Attribution (CC BY) España 3.0.

Recibido: 15/12/2016. Aceptado: 10/07/2017

\section{Abstract}

Non-renewable fossil fuels made possible what has been called the modern world. Given that non-renewable resources are increasingly becoming more and more difficult and costly to extract, the transition to renewable energy sources is a crucial task. In order to frame possible avenues towards responsible innovation and transition in democratic societies, this paper will built on the concept of "earthly experiments" to highlight some of the conditions and processes that may foster responsible innovation and learning processes. This is done by building on empirical examples from the establishment of geothermal energy systems. The paper will then unfold the sociological notion of earthly experiments in order to critically highlight on what this could mean to democratically constitute citizens as part of "science" thus conceived. The paper will show how such experimental processes deal with questions of acceptability and democratic governance, and how the political limits of experimentality are rendered visible and manageable. We show to what extent "experiments in the wild" are able to cope with increasing uncertainty and the unavoidable ignorance that complicate conditions for the transition towards renewable energy, but that may also open up new paths towards the engagement of citizens in processes of technoscientific exploration.

\section{KEYWORDS}

Engagement; Experimentation; Renewable energy; Sustainability. 


\section{INTRODUCCIÓN: EXPERIMENTOS, ENERGía Y PARTICIPACIÓN}

Los combustibles fósiles no-renovables hicieron posible el mundo moderno. Ninguna concepción de modernidad podría haber hecho sentido sin considerar la producción de energía como parte central de lo que ha sido llamado "civilización industrial". Este cambio, desde la agricultura hacia formas de producción basadas principalmente en combustibles fósiles, el que ha sido también referido como "el gran experimento" (Blumler 2008).

Dado que los recursos no-renovables en los que fue basado este experimento están siendo cada vez más difíciles y costosos de extraer, puede decirse con seguridad que el próximo gran experimento será la transición hacia recursos renovables. Después de todo, nuestra civilización no puede seguir existiendo en su forma actual sin una ininterrumpida provisión de energía. La energía, por tanto, puede ser entendida en términos sociológicos como un eje central de la forma en que las sociedades -sobre todo en nuestro industrialismo tardío (Fortun 2014) - se organizan para desarrollarse en el siglo XXI. $Y$ dado que la expansión de recursos renovables no ocurrirá en la misma proporción que la reducción en el uso de energías basadas en combustibles fósiles, la transición experimental, desde sistemas de energía basados en estos, hacia un mundo de energías renovables requerirá, no sólo un cambio tecnológico sino, también, uno sociocultural.

Basados en este diagnóstico, experimentos y cambios tecnológicos inesperados comparten ciertas similitudes cruciales. Un experimento puede ser definido en su sentido más amplio como una aventura cautelosa hacia lo desconocido o, si se prefiere, hacia el no-conocimiento ${ }^{1}$. Un experimento está deliberadamente arreglado para generar eventos inesperados: los efectos sorprendentes derivados de una propuesta experimental pueden ser vistos como la guía detrás de la producción de nuevos conocimientos y objetos (Marres 2012), a la vez que las sorpresas ayudan al experimentador a ser consciente de su ignorancia o no-conocimiento. O para decirlo de otra manera, si un experimento ha fallado y la hipótesis es falsificada dentro de los parámetros definidos por el propio experimento, entonces el experimentador fue exitoso (Stengers 2000). La diferencia crucial es que, si bien dentro de un laboratorio de investigación tales "fracasos" son bienvenidos y pueden incluso ser considerados un éxito, en el mundo fuera del laboratorio pueden ser un desastre. El fracaso de los experimentos en procesos de transición energética, de hecho, difícilmente podrían ser considerados como "éxito". En el mundo real ${ }^{2}$ los fracasos deben ser evitados.

Este artículo busca pensar qué significa ubicar lo planificadamente inesperado de los experimentos y la gobernanza del no-conocimiento en el corazón de los intentos actuales en pos de establecer un mayor uso de energías renovables (cf. Gross 2016a). Dado que reconocer la ignorancia es un elemento crucial en un proceso de experimentación, la ignorancia no puede ser entendida simplemente como la ausencia de conocimiento o anticonocimiento. Si este fuera el caso, el no-conocimiento sería evaluado como una condición indeseable. Sin embargo, investigaciones de varias disciplinas han comenzado recientemente a confrontar esta suposición negativa, explorando las formas en que la ignorancia puede ser más que la mera oposición al conocimiento. Tales investigaciones han demostrado que la ignorancia tiene una vida social y política propia (para una visión general ver Smithson 2015). Ellas han señalado que en muchas áreas de la vida social los individuos comúnmente necesitan actuar a pesar de la (algunas veces) bien definida ignorancia, o lo que George Simmel llamó no-conocimiento (Nichtwissen), a posibilidad de devenir consciente de la propia ignorancia (cf. Gross 2012). A diferencia de la noción de ignorancia, el noconocimiento refiere a lo que, si bien no se sabe, está razonablemente bien definido. El no-conocimiento constituye una forma más precisa de lo desconocido $y$, por lo tanto, puede ser empleado cuando se describe cómo se toman las decisiones que tienen un resultado incierto. También puede señalar aquellos mecanismos de control respecto a lo que debiera (o no debiera) ser conocido. El no-conocimiento será, por tanto, referido de esta manera particular cuando se discutan las estrategias experimentales involucradas en el avanzar hacia una sociedad post-combustibles fósiles y (ojalá) de energías renovables, conjuntamente con las inevitables brechas de conocimiento que esto implica.

La perspectiva del mundo como laboratorio y de la necesidad de experimentos realizados "a la intemperie" se correlaciona con la observación contemporánea de que muchos de los riesgos implicados en los procesos de investigación están siendo crecientemente desplazados hacia la sociedad, causando que los ciudadanos sean parte de experimentos de gran escala (cf. Muniesa y Callon 2007; Shapo 2009; Stilgoe 2015; van de Poel 2016). Aunque los experimentos siempre involucran a un "público" para la validación de sus resultados (Shapin and Schaffer 1989), los experimentos llevados a cabo a escala societal radicalizan el descentramiento del laboratorio, en tanto espacio privilegiado de la ciencia. Tal perspectiva sugiere que el experimento de laboratorio puede ser entendido como una extensión subordinada del experimento, el cual puede desplegarse en el mundo real. En lugar de excluir el exterior y asegurar el control de las condiciones de frontera, un experimento en el mundo real puede ser útil sólo cuando está abierto a nuevas sorpresas: estas deben ser entendidas como un elemento clave del experimento que acontece en el mundo real. Esto, argumentamos, 
abre importantes reflexiones sobre cómo los experimentos a la intemperie articulan una nueva relación entre ciencia y sociedad; esto es, hasta qué punto se presentan como un particular modo de participación pública (Lezaun et al. 2016). En la medida en que los experimentos dibujan sus propios parámetros de control (Muniesa y Callon 2007; Tironi 2014), la cuestión acerca de qué es parte del experimento, y por consiguiente qué está dentro o fuera del espacio donde el experimento toma lugar, es algo a definir durante el propio curso del proceso experimental. Es decir, la apertura del experimento hacia, en y con la sociedad, lejos de ser una anomalía, tendría que ser considerado como parte de un experimento normal, si esa apertura es parte de su configuración. En este sentido, los experimentos en el mundo real son experimentos en toda regla, estableciendo una continuidad epistemológica $-y$ no de ruptura-- con los experimentos de laboratorio.

Es importante notar que lejos de ser una excepción, los experimentos fuera-del-laboratorio han proliferado en las últimas décadas (Lezaun y Millo 2006; Guggenheim 2012). La relación entre esta multiplicación y la lógica de "testeo permanente" propia de ciertos nichos de mercado ha traído consigo importantes críticas; particularmente, a la relación entre "experimentalidad" y las formas de gobernanza del capitalismo tardío (Karvonen y van Heur 2014). La "experimentalidad" puede suponer, como veremos más tarde, un argumento "científico" para realizar intervenciones a escala masiva y cuyo valor económico radica, precisamente, en la captura de la "oportunidad" en base a altos grados de riesgo sin ningún tipo de principio precautorio.

Con el fin de mostrar cómo es posible el despliegue de estos experimentos en el mundo real, tomamos el caso de la energía geotermal. Buscamos ilustrar, en base a este caso, cómo las prácticas experimentales se despliegan en distintos lugares y momentos; y cómo gran parte de la investigación realizada en el laboratorio se articula sólo cuando el experimento está en su etapa final y se ha vuelto aparente qué tipo de investigación es requerida. El caso es relevante porque, dada la relativa novedad de la energía geotermal como fuente de energía en muchos países, es un campo caracterizado por inevitables desconocimientos e incertidumbres. Nuestro argumento avanzará de la siguiente manera. En un primer momento vamos a sugerir que los experimentos conducidos fuera del laboratorio pueden ser entendidos esencialmente como una manera de hacer uso estratégico de una ignorancia bien definida. Vamos a trabajar sobre la idea de localizar los elementos inesperados de la experimentación al centro de las tecnologías geotermales. En contraste con aproximaciones que externalizan la ignorancia por medio de analizar el riesgo, sugeriremos, en un segundo momento, que los aspectos inesperados de la producción de conocimiento pueden ser analizados usando la noción de experimentalidad. En un tercer momento, discutiremos asuntos conectados con la ignorancia percibida que es transferida/relocalizada dentro de los laboratorios cerrados ${ }^{3}$. Finalmente, reflexionaremos sobre la aceptabilidad y gobernanza democrática de tales procesos experimentales, así como sobre sus límites y capacidades para lidiar con incertidumbres crecientes y la usualmente inevitable ignorancia que complica las condiciones para la transición hacia energías renovables.

\section{Riesgo, NO-CONOCIMIENTO Y EXPERIMENTOS}

El propósito principal de los conceptos de riesgo desarrollados a partir de la Segunda Guerra Mundial, ha sido hacer del futuro algo predecible por medio de cálculos probabilísticos. La idea fue presentar el futuro como una entidad dócil para la planeación e intervención humana (Porter 1996). Entendido de este modo, el riesgo puede ser visto como una manera de racionalizar un futuro incierto (Callon et al. 2009). Evaluaciones de futuro clásicas asumen que las probabilidades de ocurrencia de un evento, en un área dada, y bajo condiciones establecidas, son conocidas; mientras que la incertidumbre es incalculable. Esta distinción fue popularizada por Frank Knight (1921). En estos términos, lidiar con la ignorancia difiere de ampliar o limitar los riesgos, dado que el riesgo de ocurrencia de un evento presupone el conocimiento, tanto del carácter del evento que podría ocurrir, como de la probabilidad de que este efectivamente ocurra (cf. Gross 2016b; Hansson 2009).

Al margen de que existan muchas otras nociones de riesgo, aquí hipotetizamos acerca de que cuando los científicos o la gente común hablan acerca del riesgo, en muchos casos sería empírica y teóricamente más útil y significativo enmarcar sus decisiones, declaraciones y prácticas en términos de diferentes tonos de ignorancia. En otras palabras, es de esperar que muchas de las cosas sobre las que hablan los tomadores de decisiones sean desconocidas para ellos, y que por lo tanto sea más importante para el análisis empírico detenerse en ellas que enfocarse en aquellas cosas o fenómenos que aparecen como conocidos. Esta posición se separa de la visión que percibe a la ignorancia como necesariamente determinada, y por el contrario, apunta hacia desafíos más amplios y (en algunos casos) le da méritos al no-conocimiento así como a las formas en que la ignorancia podría servir incluso como un recurso productivo. Esta idea puede ser fácilmente asociada con la noción de los experimentos que toman lugar en el mundo afuera de la esfera tradicional de la ciencia.

Una importante tradición dentro de los estudios en ciencia, tecnología y sociedad (CTS) ha ubicado a los experimentos y la experimentalidad en el cora- 
zón de su agenda de investigación. Si bien en un comienzo estas investigaciones se centraban en los experimentos científicos, los estudios CTS se extendieron hacia la lógica experimental en una variedad de espacios sociales. Aquí se puede incluir una amplia gama de trabajos, incluyendo estudios sobre los experimentos psicológicos (Lemov 2005; Lezaun, Muniesa y Vikkelsø 2013), el análisis de métodos de encuesta de opinión pública (Osborne y Rose 1999; Lezaun 2007), o el hazlo-tú-mismo como modo de experimentación urbana (Estalella y Sánchez-Criado 2015; Sánchez-Criado et al. 2015). Además, la intersección de los estudios CTS con la sociología económica ha dado lugar a múltiples estudios sobre el papel de la experimentación en la economía y en la construcción o simulación de mercados (Muniesa 2014). Este compromiso con los "experimentos sociales" ha implicado generalmente una ampliación de la categoría de lo experimental para abarcar cualquier esfuerzo explícito de configurar un aparato para hacer analizables los fenómenos sociales y, por lo tanto, abrir estos fenómenos a la intervención.

Para efectos de nuestro caso, hay tres elementos de esta lógica experimental que deben ser resaltados. Primero, que los experimentos, del tipo que sean, han puesto de manifiesto la mediación de objetos, cosas y aparatos en la producción de conocimiento: los estudios CTS han demostrado la importancia de esta maquinaría en la producción de conocimiento válido (Latour 1982; Marres 2012). En segundo lugar, se hizo evidente que en el transcurso de los experimentos las construcciones teóricas eran rápidamente superadas por la productividad de "hechos" de los aparatos experimentales. Es decir, que los experimentos se entendían mejor como prácticas altamente coreografiadas, cuyo desempeño, si resultaba exitoso, producía sorpresas (Rheinberger 1997).

Por último, los experimentos llaman la atención por sus particulares prácticas de demostración. La organización de exhibiciones controladas especialmente diseñadas para convencer a colegas y al público en general de la existencia de entidades y fenómenos experimentales recién descubiertos es un elemento crítico del experimento (Shapin y Schaffer 1986). Es decir, los estudios CTS han mostrado que la producción de conocimiento "en el laboratorio" por un lado, y su comunicación a públicos relevantes, por el otro, estaban mucho más estrechamente conectados de lo que generalmente se reconoce. Lo importante es notar que las demostraciones científicas no fueron solo ocasiones en las que se validaba el conocimiento experimental, sino también en las que el público se construía: el experimento requiere de una contraparte "cívica" que debe ser constituida como tal. Así, la dramaturgia de las demostraciones experimentales se convirtió en un sitio estratégico para elaborar los fundamentos probatorios de las di- ferentes culturas políticas experimentales (Shapin y Schaffer 1986; Ezrahi 1990).

Lo que se quiere enfatizar es que los públicos son producidos por las técnicas movilizadas para su participación. La participación del público, como un escenario materialmente situado y constituido, reproduce el proceso inventivo de experimentos de laboratorio. Así como los experimentos en física o biología provocan la existencia de los neutrinos o bacterias que manipulan (Latour 1993), las metodologías utilizadas en los procesos participativos -desde los grupos focales hasta los referendos en línea a las asambleas comunitarias- no se limitan a atraer a los públicos a una arena interactiva. Estas metodologías también ayudan a constituirlas en base a sus procedimientos de selección, comunicación, organización y sistematización (Irwin 2001; Girard y Stark 2007; Lezaun y Soneryd 2007; Michael 2009; Felt y Fochler 2010; Horst e Irwin 2010; Marres y Lezaun 2011; Marres 2012). Como afirman Braun y Schultz (2010: 406), en los ejercicios de participación pública, el público "nunca existe inmediatamente, sino que es inevitablemente el resultado de procesos de nomenclatura y enmarque, de una puesta en escena, de la selección y del establecimiento de prioridades, atribuciones, interpelaciones, categorizaciones y clasificaciones".

Profundizando en esta perspectiva, y con el fin de poder actuar dentro de un arreglo experimental, los actores involucrados necesitan acordar respecto a lo que no se conoce, a cómo establecerán la validez de lo que desde esa ignorancia se descubra, y tomar esto en consideración para planeamientos futuros -incluyendo la relación con el público. Los actores necesitan decidir actuar a pesar de la (algunas veces) bien definida ignorancia o lo que llamaremos 'no-conocimiento' para indicar la simetría entre lo que se conoce y lo que no se conoce. Por lo tanto, el objetivo es, por un lado, especificar la ignorancia de modo que pueda ser usada de una forma significativa y constructiva, y por el otro, establecer los parámetros de lo "significativo" para que todo lo nuevo que emerja con y desde la ignorancia sea parte del arreglo experimental. Mientras el tener fe en el control total y en el conocimiento completo de sistemas ecológicos y procesos sociales implica la habilidad de actuar solo cuando todo es conocido de antemano, la "aproximación experimental" hace posible acomodar factores diferentes en la presencia de gradientes de no-conocimiento.

Mientras que la experimentación solía ser un término que no tenía una connotación positiva clara cuando refería al aprendizaje en las políticas públicas, investigaciones recientes parecen detectar un cambio hacia un tipo más experimental de regulación política y toma de decisiones, a veces refiriendo a un nuevo modo de gobernanza en una sociedad experimental (cf. Gross 2010; Overdevest et al. 2010; 
Evans et al. 2016). Esto no impide, como ya se dijo, que la idea de experimentación tenga que ser tomada con cautela. Primero, porque si bien una verdadera aproximación experimental necesita ser entendida como una que conscientemente se mueva hacia lo desconocido por medio de una especificación de qué es lo no-conocido (por ejemplo, construyendo hipótesis) tan claramente como sea posible y luego utilizando los inevitables errores o contratiempos como base del aprendizaje, esta configuración no asegura salirse del cientificismo objetivista sobre el que suelen descansar los proyectos tecnocráticos. Dicho de otra manera, para que el reconocimiento de la ignorancia provea un verdadero ímpetu para nuevos conocimientos, se requiere que el experimento vaya más allá de las maneras usuales -hipótesis, falsificaciones, tesis- con que las ciencias han configurado su aproximación experimental.

En la próxima sección esbozaremos cómo los actores lidian con no-conocimientos ineludibles y eventos sorpresivos mientras descienden a través de las capas geológicas de la tierra.

\section{LA GEOLOGía deL SUBSUELO COMO FUENTE DE ENERGÍAS RENOVABLES}

A pesar de que el uso de la energía geotermal representa solo una pequeña parte en la transición de muchos países desde los combustibles fósiles y/o nucleares hacia formas renovables de producción de energía, su contexto experimental puede ser visto como un modelo para experimento más amplios hacia la sustentabilidad.

Algunas de las incertidumbres involucradas en el aprovechamiento de la energía geotermal son especialmente relevantes cuando se desciende hasta cinco kilómetros bajo la corteza terrestre buscando la captura de reservas energéticas mediante nuevas tecnologías de perforación. Después de todo, para la mayoría de los pozos profundos no hay datos preexistentes en los que basar un modelo confiable (Vienken et al. 2014). En este espíritu, un popular libro de ciencias sobre energías renovables argumenta sin rodeos que en la localización de sitios adecuados de fuentes de energía geotermal no existe manera de medir con precisión el verdadero potencial. Por lo que la inversión tiene que necesariamente especular, y esto aumenta el riesgo financiero (DeGunther 2009: 214). En este sentido, el movimiento hacia adelante y hacia abajo en la ingeniería geotérmica puede ser realizado solo bajo condiciones de no-conocimiento, donde la experiencia, la intuición y tal vez un adecuado trabajo de suposición pueden ser cruciales. Más aún, la energía geotermal es distinta a cualquier otro tipo de fuente de energía porque al comienzo de un proyecto la mayor fuente de no-conocimiento son los costos de inversión. La fase de exploración y el testeo de perforación están basa- dos en acercarse al éxito -o de hecho, hacia el fracaso- con cautela. Después de una etapa de investigación inicial (generalmente requiriendo de estudios geológicos conducidos por la compañía involucrada pero más frecuentemente involucrando datos disponibles solo en la literatura) el titular del proyecto "tendrá que decidir si continúa o no desarrollando el proyecto y si asume o no los riesgos de la siguiente fase" (Bloomquist et al. 2013: 253). Esto se debe, entre otras razones, a que "la modelación computacional que cuantifique adecuadamente el costo alternativo de esta fuente de incertidumbre en relación a otros recursos competitivos aún no ha sido desarrollada" (Íbid.: 266). En consecuencia, Giardini (2009: 849) resume la situación actual en utilización de la energía geotermal en los siguientes términos: "Desde su inicio, los proyectos de EGS (sistema geotermal aumentado) ${ }^{4}$ necesitan ser pensados en tanto proyectos pilotos que involucran no-conocimientos científicos así como aventuras comerciales con riesgos financieros y tecnológicos". A pesar de muchas incertidumbres y no-conocimientos -los que incluyen la contaminación de aguas subterráneas debido a las perforaciones, la cuestión de los compromisos a largo plazo, y otras dificultades tecnológicas- los prospectos de adquirir nuevo conocimiento van en aumento, realizando investigación en nuevas tecnologías de control remoto y herramientas de simulación para ser utilizadas en substratos geológicos.

\section{ESTABLECIENDO LABORATORIOS DE SITIO DURANTE EL EXPERIMENTO}

Consideremos ahora dos ejemplos de levantamiento de un laboratorio después de que el experimento ya ha empezado. El primer ejemplo es delineado a partir del contexto de las prácticas de perforación geotermal y el testeo asociado del comportamiento de los componentes de los fluidos geotermales realizado en laboratorios separados del experimento en el mundo real. El segundo ejemplo es delineado a partir de un grupo focal que involucró a las partes interesadas en proyectos geotermales -un ejemplo de relocalización de sujetos humanos en un escenario semejante a un laboratorio- ${ }^{5}$.

En muchas operaciones de perforación de pozos geotermales el aparataje del taladro (como en el de las perforaciones de gas y petróleo) está repleto de dispositivos electrónicos que controlan la dirección de este. Sin embargo, estos dispositivos y herramientas de operación de tecnología avanzada solo pueden operar correctamente bajo los 150 grados Celsius. Muchas reservas de energía geotérmica en países sin condiciones óptimas en términos de fuentes geotérmicas, como Hawaii o Islandia, solo se convierten en una opción válida a temperaturas por encima de los 150 grados. En casos como estos, la perforación es usualmente realizada con correcciones hechas ad hoc durante la operación. Estas 
involucran decisiones con respecto a determinar cuál es la posición exacta del instrumento de medición en el aparataje de perforación (cercano a la parte final o bien más allá), cuándo la medición exacta debe ser realizada (dado que cada medición no es solo un factor de costos sino (y también) un asunto importante que afecta la fiabilidad general de los datos), y. por último, regular el suministro de energía a los instrumentos de medición. Además, las tecnologías de perforación, que han sido adaptadas desde las perforaciones de gas y petróleo, necesitarán manejar volúmenes mucho mayores de líquidos en comparación con las faenas de gas y petróleo, de modo que el diámetro de las brocas requiere ser bastante mayor. Los equipos de perforación funcionando en altas temperaturas son, por tanto, objeto de una alta tasa de fracaso (Glassley 2010: 135-152). En el libro de bolsillo Las buenas prácticas para la perforación geotérmica el problema se frasea de la siguiente manera: "Las dificultades inherentes a la perforación direccionada son agravados en los pozos geotérmicos porque tanto las herramientas electrónicas usadas en el control y seguimiento de la trayectoria del pozo como los elementos elastoméricos de los motores son susceptibles de estropearse en altas temperaturas" (Finger y Blankenship 2010: 46).

Científicos e ingenieros suelen destacar que los procesos basados en la experiencia e intuición involucrados en tales decisiones permiten ajustar el procedimiento de perforación en un área de la operación, pero provocando cambios en otras. El aprovechamiento de la energía geotérmica puede ser, por lo tanto, vista como desplegándose en un ámbito que está más allá de lo establecido en función de la acumulación de conocimiento existente por parte de la ciencia (lógica, por ejemplo, detrás de la validación de las publicaciones científicas a través de la revisión de pares). Los proyectos, más bien, se vuelven "exitosos" sin nadie saber exactamente por qué.

La fase de exploración de un proyecto geotermal (la etapa de prospección geológica y geofísica) y la fase de acceso que involucra la perforación y registro, son llevadas a cabo "a espaldas" de la sociedad. Los proyectos geotermales están asociados con niveles molestos de ruido así como al riesgo de actividad sísmica desencadenada por la perturbación de las rocas. Esta puede afectará edificios e infraestructuras situadas sobre el suelo (Mukuhira et al. 2013; Grimm et al. 2014) y por ello no son pocas las protestas públicas que han llevado en los últimos años a cancelar proyectos geotérmicos y a un declive general en la aceptación social y presentación mediática de esta tecnología (Stauffacher et al. 2015; Bleicher y Gross 2016).

Durante un proyecto de perforación que involucre círculos repetidos de agua geotermal, los experimentos hidráulicos son realizados en el mismo pozo con el fin de asegurar el funcionamiento a largo plazo de la reserva intervenida. Todas las mediciones son realizadas in situ con el propósito de observar el comportamiento de las zonas intervenidas. Sin embargo, solo a unos metros de distancia del experimento es usualmente necesario construir un laboratorio de sitio: un laboratorio científico, en su aspecto más clásico, para realizar investigación geotérmica detallada en caso que se encuentren descubrimientos $u$ obstáculos inesperados bajo tierra. Por ello, el experimento "terrenal" (el pozo con sus perforaciones y actividades sísmicas) no parece ser capaz de existir sin el laboratorio experimental. De hecho, todos los nuevos procesos experimentales llevados a cabo en el mundo de la ingeniería son luego escalados dentro del mundo cerrado del laboratorio. Las actividades allí realizadas incluyen la clasificación de los aspectos geológicos de los fluidos, la determinación de la composición química de estos durante las operaciones y el análisis de los gases encontrados. Otros laboratorios in situ son usados para probar técnicas de estimulación hidráulica en diferentes sedimentos y rocas volcánicas. En otras palabras, con el fin de hacer posible cualquier tipo de declaración pública acerca de la ingeniería geotermal es requisito contar con investigación científica sistemática, la que solo puede ser realizada post hoc en el laboratorio. En el caso de la perforación geotérmica, por consiguiente, el debate respecto a riesgos posibles y brechas en el conocimiento es trasladado desde el terreno al laboratorio. Esto usualmente significa, por ejemplo, que se le solicite a un grupo de ingenieros explicar al público interesado qué se sabe y qué no sobre la influencia que un experimento en el mundo real tiene sobre edificaciones residenciales cercanas o sobre el suelo y aguas subterráneas. Esto es un asunto difícil pero crucial.

Esto nos lleva al segundo ejemplo en donde las partes interesadas (ciudadanos, ingenieros, tomadores de decisiones) son llevados a otro laboratorio, también delimitado pero distinto al experimento en terreno, donde se ejecutará lo que podría denominarse un experimento en participación (Lezaun et al. 2016). Alexander Bogner (2012: 519) se ha referido a este tipo de experimentos participativos como una 'forma de participación organizada por profesionales especializados en la participación, tomando lugar bajo condiciones controladas y sin referencia a controversias públicas, demandas provenientes de la participación política, o preocupaciones individuales'. Más allá de la metodología de estos experimentos, su característica es su capacidad para performar o constituir a los participantes: estos no preexisten al evento participativo sino que son modulados en tanto 'participantes' en el proceso mismo a través de mecanismos de selección, instauración de protocolos de comunicación y delimitación de temas a discutir (Lezaun y Soneryd 2007; Braun y Schultz 2010; Tironi 2014). En concreto, nos enfocaremos en un estudio sobre involucramiento ciudadano en una pe- 
queña ciudad de Sajonia cercana a la frontera del estado alemán de Thurimgia y la República Checa en las Montañas Ore (Erzgebirge). Aquí se instaló un experimento en terreno de perforación geotérmica con el plan de construir una central de energía. Para involucrar a la ciudadanía, se llevó a cabo un laboratorio experimental en toda regla, "aislando" a los ciudadanos involucrados en una locación lejana a su vida cotidiana (un centro de conferencias en un pequeño balneario). Siguiendo la idea por la cual la construcción de una opinión válida puede ser constituida a través de un escenario experimental controlado (Lezaun 2007; Tironi 2014), el procedimiento fue diseñado para asegurar que los participantes fueran capaces de hablar y actuar libremente sin tener consecuencias sobre el experimento geotérmico mayor -del mismo modo que los laboratorios científicos suelen hacerlo cuando las consecuencias de las actividades del laboratorio no afectan a la validez ni a la operación del mismo laboratorio.

La razón para organizar este proceso participativo estaba en las grandes brechas de conocimiento por parte de la percepción pública en lo referente a la naturaleza y características del ambiente subterráneo. La invisibilidad de los procesos geotérmicos, especialmente en las profundidades del subsuelo, tiene el potencial de promover suspicacia y miedo, lo cual es usualmente visto como un obstáculo para la perforación geotérmica y la difusión de estas tecnologías -así como para la comunicación de los beneficios de estas-. Es necesario, de este modo, dar a conocer esta invisibilidad. El montaje del tipo laboratorio no es gratuito. En este, los inversores e ingenieros, en un ambiente controlado y por medio de representaciones, revelan estratégicamente cuáles son las tecnologías de perforación y cómo varían las temperaturas.

El proceso participativo en sí fue organizado por expertos de dos grandes instituciones de investigación alemanas. La invitación al laboratorio fue dada a conocer por medios locales y regionales y publicitada como un lugar donde obtener información de primera mano acerca de la perforación geotermal y la generación de energía. Los participantes que accedieron a participar no fueron seleccionados por ningún método de representatividad sino que sobre la base de quien primero llega, primero se sirve. Básicamente esto significó que todos quienes sintieran la necesidad de información o quisieran discutir asuntos relacionados y sus "puntos de vista" fueran bienvenidos. De esta manera, el laboratorio no fue levantado para pacificar protestas (a pesar de que esto podría haber sucedido) sino que por, el contrario, lo fue para observar -o movilizar (Lezaun y Soneryd 2007) - las reacciones del público frente a situaciones de incertidumbre en tal contexto.

Esta opción tuvo su costo. Un pequeño artículo en el mayor diario de Sajonia, el Freire Press (con más de 250.000 copias vendidas solo por medio de suscripciones) reportó sobre el laboratorio. El reportaje, sin embargo, apuntó directamente al carácter meramente informativo del evento. En efecto, el propósito exacto de congregar a un grupo de ciudadanos más bien fue buscar informar a la población y debatir conjuntamente sobre las incertidumbres aceptables y las no aceptables. El laboratorio, el cual duró casi un día completo, utilizó el método de grupo focal. Si bien este método ha sido recientemente destacado como una manera de proveer información específica acerca de grupos sociales, y descrito como un proceso capaz de involucrar interacciones naturales en situaciones artificiales, también ha estado bajo el escrutinio de las ciencias sociales por su configuración al modo de un "mercado de opiniones" donde se va manufacturando una "opinión colectiva” (Lezaun 2007).

En la situación participativa del tipo laboratorio aquí estudiada, los temas fueron discutidos frontalmente. Los mayores focos de discusión fueron el riesgo sísmico, la relación entre la perforación geotérmica y las tecnologías de fracking contemporáneas, los posibles incentivos económicos para la región, y el rol de la energía geotérmica dentro del plan general de establecer fuentes de energía renovable en Alemania y Europa. De este modo, y a pesar de que las intervenciones de la sociedad civil movilizada jugaron un rol importante en la discusión sobre la gobernanza de procesos ecológicos y tecnológicos, la lógica del laboratorio participativo realizado en Sajonia fue más cercana a una "invitación participativa": los participantes potenciales tuvieron que ser convencidos de participar a través de los medios locales (periódicos, avisos publicitarios, e información en paneles informativos) en una situación de laboratorio en la que le fueron asignados "un asiento en mesas redondas de discusión". O puesto de otro modo, más que una implicación a la intemperie, la participación obligó a "retirarse de la sociedad al laboratorio" (Bogner 2012: 522).

Este laboratorio, entonces, se constituyó como un espacio donde los actores interesados se sentaron juntos alrededor de una mesa y discutieron si los procesos geotérmicos en cuestión debían o no ser "testeados"6 (un término frecuentemente utilizado por los actores interesados en operaciones de perforación geotermal). Esto también incluyó explicar a los actores involucrados y a los tomadores de decisiones por qué en una cierta situación las cosas no pueden ser conocidas de mejor manera dentro de un marco de tiempo dado, lo cual requiere por contrapartida dejar en claro que es importante comenzar a trabajar en orden para no desperdiciar tiempo, y por consiguiente limitar las posibilidades de éxito.

Esto, sin embargo, es exactamente lo que suele implicar un análisis de riesgo. En otras palabras, reconocer la ignorancia no debe ser confundido con 
una falla en la experiencia o en la experticia. Más bien, debe ser entendido como un proceso normal en la producción de conocimiento experimental (Gross 2010). De más está decir, con todo, que en muchos casos la ciudadanía puede no estar interesada en estos debates y los participantes deben, como lo pone Bogner (2012: 510), ser arrastrados dentro del laboratorio para contar con sus aportes y conocimientos (ver también Tironi 2015).

Este proceso participativo, en el que los participantes tuvieron que ser seducidos a la participación dado su bajo interés, la noción de "laboratorio" toma otra dimensión: no solo la de un escenario controlado sino también la de un espacio de debate relacional pero aislado y hermético cuyos resultados (hasta aquí) no ha tenido reales consecuencias para las políticas públicas del mundo exterior. A diferencia de experimentos participativos en los que se requiere tomar decisiones urgentes y distribuir responsabilidades, el proceso aquí discutido se desenvolvió en base a escenarios potenciales o conflictos aún por ocurrir, alejando sus resultados de la posibilidad de tener efectos concretos (Tironi 2015; Lama y Tironi, en prensa). Es decir, por su baja intensidad y su naturaleza más bien informativa, la realización de este laboratorio participativo contribuyó a legitimar uno de los experimentos fuera-del-laboratorio más audaces que se hayan realizado en la zona.

Sin embargo, sin este tipo de laboratorios participativos en las sociedades democráticas los experimentos geotérmicos no podrían estar completos. Con el fin de involucrarse significativamente en tales experimentos y de lidiar con el no-conocimiento, los actores implicados necesitarían desarrollar una apertura hacia la posibilidad de redefinir sus objetivos a través de discusiones iterativas. Experimentos de este tipo tienen más probabilidades de ser llevados a cabo cuando las brechas en el conocimiento son definidas con claridad, es decir, cuando se asume que el no-conocimiento, precisamente por no ser conocido, puede hacerse aparecer durante el proceso participativo. Esto puede referir a las fases de perforación durante las cuales una evaluación debe ser realizada acerca de si algunas de las brechas de conocimiento percibidas pueden ser reducidas con mayor investigación geológica o con más datos de modelación, o si solo la estrategia de "aprendiendo mientras se hace" es concebible. En este marco, la toma de decisiones, que está basada sobre un conjunto de no-conocimientos discutidos en experimentos de participación en laboratorios, no sería considerada un mero retoque irresponsable, sino más bien estaría basada en un sentido compartido de ignorancia justificable (razonable), la cual ha sido asumida como tal y definida claramente ( $y$ tal vez incluso consensualmente acordada) de modo que el experimento pueda continuar.

\section{LLEVANDO EL EXPERIMENTO (DE VUELTA) AL LABORATORIO}

Como hemos visto con el ejemplo de la energía geotermal, la idea de que es necesario un laboratorio para entender el mundo fuera de aquel puede ser razonablemente puesta en duda (cf. Latour 1982). En muchos casos, como en el que nos convoca, parece aplicar lo contrario: un experimento parece estar allí primero, mientras que el laboratorio es determinado post-hoc. En el ámbito del uso de la energía geotermal puede indicarse que las prácticas experimentales ocurren en muchos lugares mientras que el laboratorio y los actores sociales externos a las esferas institucionales de la ciencia arriban al escenario solo después del comienzo del experimento -cuando, por ejemplo, los actores interesados son invitados a participar en situaciones de tipo laboratorio, o cuando son testeadas las particularidades de los fluidos geoquímicos-. En los experimentos fuera-del-laboratorio los peligros y conflictos suelen aparecer solo una vez que la tecnología ha sido testeada o luego de que los actores involucrados hayan sido invitados al laboratorio. Estas situaciones de incertidumbre compartida abren, en potencia, la posibilidad de aprovechar todas las capacidades de la exploración experimental.

Las conversaciones entre expertos y actores ciudadanos en contextos de laboratorio pueden por lo tanto ser entendidas como parte importante de los experimentos terrenales, tal como lo son las investigaciones de laboratorio en las que están involucrados los científicos. Ciertamente es una parte importante para el aprovechamiento de la energía geotermal y quizás también de la transición energética desde los combustible fósiles a combustibles renovables en general. Tal transición implica la alteración en la forma en que se administra, investiga y planifica la producción energética. Esto parece alentar, e incluso requerir, el desarrollo de estrategias innovadoras y la utilización de los potenciales recursos de los actores involucrados en pos de un objetivo común. Aún más, el nuevo conocimiento puede emerger desde eventos inesperados, los cuales en laboratorios definidos post-hoc pueden ayudar a especificar más aún la ignorancia, de modo que los límites y fronteras del conocer sean conscientemente tomados en cuenta cuando se actúa o planifica. Así, los experimentos en el mundo real deben ser diseñados para convivir con lo no-conocido mediante su anclaje -aunque algunas veces solo brevemente- a un laboratorio tradicional, social o de otro tipo.

Puede quedar la impresión de que, algunas veces, para legitimar un experimento fuera-del-laboratorio algunos de sus componentes críticos son trasladados al laboratorio para invisibilizarlos y dejarlos sin consecuencias. Si falla el laboratorio en un experimento en el mundo real puede que el proceso completo se detenga (por ejemplo, si los actores sociales no pueden ponerse de acuerdo respecto a una decisión, 
el análisis de carbones es inútil). En consecuencia, el necesario resguardo de la producción de conocimiento detrás de las paredes del laboratorio -ya sea para las actividades de trabajo de los actores ciudadanos o para las actividades de los científicos- sigue siendo importante. Sin embargo, la constitución y el funcionamiento de estos laboratorios clausurados se origina a partir de un proceso experimental en el mundo real que involucra a una diversidad de actores y entidades-compañías de energía geotermal, científicos, ingenieros y ciudadanos involucrados, entre otras -o lo que también se ha denominado foros híbridos (Callon et al. 2009)-. Es decir, la laboratorización sigue siendo importante, pero es dependiente de procesos mucho más amplios e inciertos en el mundo real que no pueden ser reducidos. Puesto de otra manera, el caso de la energía geotermal muestra que, en algunas situaciones, se invierte la secuencia tradicional desde la investigación de laboratorio a la aplicación en el terreno: la instalación del experimento es primero y a veces incluso independiente de un laboratorio claramente definido que solo vendrá al escenario posteriormente.

Los inevitables no-conocimientos e incertidumbres presentes en tales experimentos son relocalizados fuera en "laboratorios" correspondientes. Dependiendo del tipo de incertidumbre y no-conocimiento, estos pueden ser laboratorios científicos o una participación ciudadana laboratorizada con los actores involucrados -o ambos-. Se puede decir, entonces, que la investigación sobre energía alternativa en ambientes subterráneos, como las discutidas en este artículo, muestra una tendencia donde los procesos de investigación comienzan en el mundo real y los problemas que surgen son desplazados al mundo más cerrado del laboratorio (y sus varias configuraciones), y no al revés. Los experimentos del mundo real proveen de una justificación para las actividades de laboratorio. Bajo esta perspectiva, los experimentos de laboratorio son micro variantes post-posicionadas de los experimentos a la intemperie. Así entendida, la producción siempre riesgosa de nuevo conocimiento por medio de la experimentación en el mundo real promueve una práctica que cambia el contexto del descubrimiento científico.

\section{PANORAMA: DEMOCRACIA Y EXPERIMENTOS EN EL MUNDO REAL}

Cuando un nuevo conocimiento es adquirido, este suele llevar a la percepción de nuevos horizontes acerca de lo que no es conocido. Sin embargo, cuando se refiere a planificar y manejar procesos de restauración o revitalización de paisajes alterados industrialmente, es aún común entre policy makers y científicos asumir que las decisiones deben ser tomadas sobre la base de conocimientos científicos confiables. Solo estos "hechos" conocidos y observables son explícitamente incluidos en la evaluación de decisiones políticas y de riesgo. Esto lleva a establecer una brecha entre la retórica oficial, por un lado, y la situación de los actores en terreno por el otro, para quienes se vuelve casi la norma el lidiar experimentalmente con la ignorancia.

Más aún, si aparece como significativo el caracterizar a las sociedades contemporáneas modernas como dirigiéndose hacia más experimentalidad, y si la experimentalidad se está convirtiendo en un personaje definido de la sociedad del siglo XXI (cf. Gross 2010; Hodson y Marvin 2016; Lezaun et al. 2016; Shapo 2016), entonces la experimentación pública y el reconocimiento del no-conocimiento están dispuestos para volverse más importantes que nunca. Esto significaría alejarse de la orientación hacia experiencias previas y extrapolaciones históricas en la naturaleza, tecnología y sociedad (por ejemplo, el análisis de riesgo) y en cambio moverse hacia la prospectiva y nociones más complejas de lo conocido y lo no-conocido. Esto puede estar en línea con lo que ha sido llamado imperativo proaccionario (More 2005; Fuller y Lipinska 2014), pero alberga algunos desafíos que necesitan ser aclarados: los actores involucrados necesitan acordar respecto a que no hay otros medios razonables disponibles (con respeto al tiempo, financiamiento y resultados esperados) para ganar conocimiento respecto a un cierto asunto que parezca relevante (cf. Gross 2010). Con todo, esto no será siempre posible, y por tanto las posibilidades de detener un experimento tienen que ser clarificadas de antemano. Más aún, y esto puede ser el asunto más problemático, una distribución justa de los beneficios y peligros necesita ser definida. En una versión del experimento en el mundo social anclada democráticamente, esto también significa que la mayor cantidad de gente posible necesita beneficiarse de él. Nuevamente, esto puede sonar realizable en teoría, pero un amplio abanico de distintas perspectivas son necesarias para ofrecer al menos una base para entender los beneficios potenciales en la práctica. La tarea, entonces, se convierte en una de formulación experimental que permita que las perspectivas públicas sean incorporadas al diseño y formen parte del camino imaginado.

A pesar del discurso sobre la experimentalidad en algunas áreas de investigación y política pública, las retóricas oficiales promueven mayor certidumbre y más medidas de seguridad. Después de todo, el principio de precaución está bien establecido en el pensamiento cotidiano. Desde esta perspectiva, la toma de decisiones basadas en el no-conocimiento sería irresponsable, lo cual ciertamente es una posibilidad, pero eso no invalida que este sea un hecho inevitable que debe ser incluido en las declaraciones oficiales. A pesar de esto, la toma de decisiones en el mundo real, y sobre todo dentro de burocracias estrictamente reguladas, parece promover paradójicamente un compromiso positivo con la ignorancia y la sorpresa. En una 
sociedad donde la práctica científica define el espacio legal de fracaso, sus contratiempos y la existencia y valor del riesgo, la producción de no-conocimiento experimental parece aumentar en arreglos espaciales y temporales claramente definidos.

Esto, por supuesto, puede también invitar a las corporaciones o actores individuales con motivos egoístas a usar la incertidumbre inherente de la práctica experimental como una excusa para asegurar una salida si las acciones que promueven resultan ser desastrosas. Esto puede ser una razón de por qué las estrategias experimentales son más frecuentemente encontradas en proyectos de innovación e ingeniería tales como los que encontramos en algunas operaciones de energía renovable. En este sentido, la experimentalidad no es una idea romántica de gradualismo, de atravesar la confusión, o un tipo de administración adaptativa donde los actores se ajustan pasivamente a cambiantes condiciones externas; por el contrario, esta demuestra de una manera clara que lidiar con el no-conocimiento puede convertirse en una realidad, no por elección sino por la necesidad de un futuro sustentable. Es tentador especular con que, al nivel de gobiernos locales o regionales, las estrategias experimentales prosperan de mejor

\section{NOTAS}

1. Del término en inglés nonknowledge.

2. En lo que sigue utilizamos "mundo real", "terrenal" y "fuera-del-laboratorio" como sinónimos. Por "mundo real" no implicamos una distinción ontológica (como si lo que ocurriese en un laboratorio no fuese real) sino una empírica: todo aquello que sucede al otro lado de la reclusión y el asilamiento propio de los laboratorios experimentales.

3. Los casos de este artículo están basados en dos proyectos interdisciplinarios de conducidos por el Helmholtz Centre of Environmental Research (UFZ) en Leipzig, Alemania. El material que aquí se presenta está basado en notas de campo y entrevistas realizadas por Matthias

\section{RefERENCIAS BibLIOGRÁficas}

Bleicher, A. y M. Gross. 2015. "User Motivation, Energy Prosumers and Regional Diversity: Sociological Notes on using Shallow Geothermal Energy". Geothermal Energy 3(1): 1-12, [art 12]. http://dx.doi.org/10.1186/ s40517-015-0032-6

Bleicher, A. y M. Gross. 2016. "Geothermal Heat Pumps and the Vagaries of Subterranean Geology: Energy Independence at a Household Level as a Real World Experiment". Renewable \& Sustainable Energy Reviews 64: 279-288. http://dx.doi.org/10.1016/j.rser.2016.06.013

Bloomquist, G, J. Lund y M. Gehringer. 2013. "Geothermal Energy". Pp. 245-273 en The World Scientific Handbook of Energy: Materials and Energy (vol. 3), editado por G. Crawley and M. Hackensack, NJ: World Scientific Publishing.

Blumler, M. 2008. "The Fossil Fuel Revolution: A Great, and Largely Unrecognized, Experiment". Pennsylvania Geographer 46 (2): 3-21. manera cuando existen regulaciones estatales estrictas en lugar de proveer a los "pro-accionarios" con la libertad de experimentar y también de fallar.

Al fin de cuentas, un prerrequisito importante para el "fracaso exitoso" a través de la experimentación es que los actores involucrados estén preparados para tomar decisiones a pesar de la existencia de ignorancias. También necesita de debate público y de la existencia de ciudadanos preparados para la transformación y la disrupción. Esto conlleva una cultura de reconocimiento del no-conocimiento, de la anti-culpa o recriminación, y de clarificación de los beneficios de moverse hacia lo no conocido. Esto puede ser difícil de encontrar en la retórica oficial pero, como debieran haber indicado los ejemplos discutidos en este artículo, en distintas culturas prácticas estos parecen estar vivos y activos.

\section{AgradeCIMIENTOS}

Manuel Tironi agradece el apoyo del FONDECYT 1150319, del Núcleo Milenio de Investigación en Energía y Sociedad (NUMIES) y del Centro de Investigación para la Gestión Integrada de Riesgo de Desastres (CIGIDEN), Conicyt/FONDAP/15110017
Gross en visitas a sitios de perforación y a través de grupos de trabajo en energía geotermal, discusiones en grupos focales y reuniones con tomadores de decisiones y actores involucrados. Para mayor detalle y otros resultados de este proyecto de investigación ver Bleicher y Gross $(2015,2016)$ como también Gross (2013, 2015, 2016a).

4. N. de T.: Por sus siglas en inglés 'Enhanced Geothermal System'.

5. Esta sección está parcialmente basada en Gross (2016a).

6. Del término en inglés tried out.
Bogner, A. 2012. "The Paradox of Participation Experiments". Science, Technology \& Human Values 37 (5): 506-527. http://dx.doi.org/10.1177/0162243911430398

Braun, K, y S. Schultz. 2010. “'... a certain amount of engineering involved': Constructing the public in participatory governance arrangements". Public Understanding of Science 19(4): 403-419. http://dx.doi. org/10.1177/0963662509347814

Callon, M, P. Lascoumes y Y. Barthe. 2009. Acting In An Uncertain World. Cambridge, MA: The MIT Press.

DeGunther, R. 2009. Alternative Energy for Dummies. Hoboken, $\mathrm{NJ}$ : Wiley.

Estalella, A. y T. Sánchez-Criado. 2015. "Experimental Collaborations: An Invocation for the Redistribution of Social Research". Convergence: The International Journal of Research into New Media Technologies 21 (3): 301-305. http://dx.doi. org/10.1177/1354856515579839 
Evans, J., A. Karvonen and R. Raven. 2016. The Experimental City. London: Routledge.

Ezrahi, Y. 1990. The Descent of Icarus: Science and the Transformation of Contemporary Democracy. Cambridge, MA: Harvard University Press.

Felt, U. y M. Fochler. 2010. "Machineries for Making Publics: Inscribing and De-scribing Publics in Public Engagement". Minerva 48: 219-238. http://dx.doi.org/10.1007/ s11024-010-9155-x

Finger, J. y D. Blankenship. 2010. Handbook of Best Practices for Geothermal Drilling. Albuquerque, NM: Sandia National Laboratories.

Fortun, K. 2014. "From Latour to late industrialism". Hau 4: 309-329. http://dx.doi.org/10.14318/hau4.1.017

Fuller, S. y V. Lipinska. 2014. The Proactionary Imperative: A Foundation for Transhumanism. Houndmills, UK: Palgrave Macmillan.

Giardini, D. 2009. "Geothermal Quake Risks Must be Faced". Nature 462: 848-849. http://dx.doi.org/10.1038/462848a

Girard, M. y D. Stark. 2007. "Socio-technologies of Assembly: Sense making and Demonstration in Rebuilding Lower Manhattan". Pp. 145-176 en Governance and Information:The Rewiring of Governing and Deliberation in the 21st Century, editado por D. Lazer and V. Mayer-Schoenberger. New York y Oxford: Oxford University Press.

Glassley, W. 2010. Geothermal Energy: Renewable Energy and the Environment. London: CRC Press.

Grimm, M, I. Stober, K. Thomas y P. Blum. 2014. "Schadensfallanalyse von Erdwärmesondenbohrungen in BadenWürttemberg". Grundwasser 19(4): 275-86. http:// dx.doi.org/10.1007/s00767-014-0269-1

Gross, M. 2010. Ignorance and Surprise: Science, Society, and Ecological Design. Cambridge, MA: MIT Press.

Gross, M. 2012. "'Objective Culture' and the Development of Nonknowledge: Georg Simmel and the Reverse Side of Knowing". Cultural Sociology 6(4): 422-437. http:// dx.doi.org/10.1177/1749975512445431

Gross, M. 2013. "Old Science Fiction, New Inspiration: Communicating Unknowns in the Utilization of Geothermal Energy". Science Communication 35 (6): 810-818. http://dx.doi.org/10.1177/1075547012469184

Gross, M. 2015. "Journeying to the Heat of the Earth: From Jules Verne to Present-Day Geothermal Adventures". Engineering Studies 7(1): 28-46. http://dx.doi.org/10.1 080/19378629.2014.997738

Gross, M. 2016a. "Give me an Experiment and I will raise a Laboratory". Science, Technology \& Human Values 41 (4): 613-634. http://dx.doi.org/10.1177/0162243915617005

Gross, M. 2016b. "Risk as Zombie Category: Ulrich Beck's Unfinished Project of the Nonknowledge Society". Security Dialogue 47(5): 386-402. http://dx.doi. org/10.1177/0967010616645020

Guggenheim, M. 2012. "Laboratizing and de-laboratizing the world changing sociological concepts for places of knowledge production". History of the Human Sciences 25.1: 99-118. http://dx.doi. org/10.1177/0952695111422978

Hansson, S. 2009. "From the Casino to the Jungle: Dealing with Uncertainty in Technological Risk Management". Synthese 168(3): 423-432. http://dx.doi.org/10.1007/ s11229-008-9444-1

Hodson, M. y S. Marvin. eds. 2016. Reftrofitting Cities: Priorities, Governance, and Experimentation. London: Routledge.

Horst, M. y A. Irwin. 2010. "Nations at ease with radical knowledge: on consensus, consensusing and false consensusness". Social Studies of Science 40(1): 105126. http://dx.doi.org/10.1177/0306312709341500

Irwin, A. 2001. "Constructing the scientific citizen: Science and democracy in the biosciences". Public Understanding of Science 10(1): 1-18. http://journals.sagepub.com/doi/ abs/10.3109/a036852?journalCode=pusa

Karvonen, A. y B. van Heur. 2014. "Urban laboratories: experiments in reworking cities". International Journal of Urban and Regional Research 38(2): 379-92. http:// dx.doi.org/10.1111/1468-2427.12075

Knight, F. 1921. Risk, Uncertainty, and Profit. Boston, MA: Mifflin.

Lama, J. y M. Tironi. En prensa. "Distributing obligations, performing publics: responsible citizens in post-disaster engagement". Qualitative Sociology.

Latour, B. 1982. Science in Action: How to Follow Scientists and Engineers through Society. Cambridge, MA: Harvard University Press.

Latour, B. 1993. The Pasteurization of France. Cambridge, MA: Harvard University Press.

Lemov, R. 2005. World as Laboratory: Experiments with Mice, Mazes, and Men. New York: Macmillan.

Lezaun, J. 2007. "A Market of Opinions: The Political Epistemology of Focus Groups". Sociological Review 55 (s2): 130-51. http://dx.doi.org/10.1111/j.1467954X.2007.00733.X

Lezaun, J. y Y. Millo. 2006. "Regulatory experiments: GM crops and financial derivatives on trial". Science and Public Policy 33(3): 179-190. http://dx.doi. org/10.3152/147154306781779046

Lezaun, J, F. Muniesa y S. Vikkelsø. 2013. "Provocative containment and the drift of social-scientific realism". Journal of Cultural Economy 6(3): 278-293. http://dx.doi.or $\mathrm{g} / 10.1080 / 17530350.2012 .739972$

Lezaun, J. y L. Soneryd. 2007. "Consulting Citizens: Technologies of Elicitation and the Mobility of publics". Public Understanding of Science 16(3): 279-297. http://dx.doi. org/10.1177/0963662507079371

Lezaun, J, N. Marres y M. Tironi. 2016. "Experiments in Participation". Pp. 195-222 en Handbook of Science and Technology Studies (vol. 4), editado por C. Miller, E. Smitt-Doer, U. Felt y R. Fouche. Cambridge: MIT Press.

Marres, N. 2012. Material Participation. Technology, the Environment and Everyday Publics. London: Palgrave Macmillan.

Marres, N. y J. Lezaun. 2011. "Materials and devices of the public: an introduction". Economy and Society 40(4): 489509. http://dx.doi.org/10.1080/03085147.2011.602293

Michael, M. 2009. "Publics performing publics: of PiGs, PiPs and politics". Public Understanding of Science 18(5): 617631. http://dx.doi.org/10.1177/0963662508098581

More, M. 2005. "The Proactionary Principle". Disponible en: http://www.maxmore.com/proactionary.html [consultado el 26/08/2016].

Mukuhira, Y, H. Asanuma, H. Niitsuma y M. Häring. 2013. "Characteristics of Large-Magnitude Microseismic Events Recorded During and After Stimulation of a Geothermal Reservoir at Basel, Switzerland". Geothermics 45 (1): 1-17. http://dx.doi.org/10.1016/j.geothermics.2012.07.005

Muniesa, F. 2014. The Provoked Economy: Economic Reality and the Performative Turn. London: Routledge.

Muniesa, F. y M. Callon. 2007. "Economic experiments and the construction of markets". Pp. 163-189 en Do economists make markets? On the Performativity of Economics, editado por D. MacKenzie, F. Muniesa y L. Siu. Princeton, NJ: Princeton University Press. 
Osborne, T. y N. Rose. 1999. "Do the Social Sciences Create Phenomena? The Example of Public Opinion Research". British Journal of Sociology 50(3): 367-96. http://dx.doi.org/10.1111/j.1468-4446.1999.00367.x

Overdevest, C, A. Bleicher y M. Gross. 2010. "The Experimental Turn in Environmental Sociology: Pragmatism and New Forms of Governance". Pp. 279-294 en Environmental Sociology: European Perspectives and Interdisciplinary Challenges, editado por M. Gross y $\mathrm{H}$. Heinrichs. Heidelberg: Springer.

Porter, T. 1996. Trust in Numbers: The Pursuit of Objectivity in Science and Public Life. Princeton, NJ: Princeton University Press.

Rheinberger, H-J. 1997. Toward a History of Epistemic Things: Synthesizing Proteins in the Test Tube. Stanford: Stanford University Press.

Sánchez-Criado, T, I. Rodríguez-Giralt y A. Mencaroni. 2015. "Care in (Critical) Making: Prototyping as a Radicalisation of Independent-Living politics". ALTER. European Journal of Disability Research. 10(1): 24-39. http:// dx.doi.org/10.1016/j.alter.2015.07.002

Shapin, S. y S. Schaffer. 1986. Leviathan and the Air-Pump: Hobbes, Boyle, and the Experimental Life. Princeton: Princeton University Press.

Shapo, M. 2009. Experimenting with the Consumer: The Mass Testing of Risky Products on the American Public. Westport, CT: Praeger.

Shapo, M. 2016. The Experimental Society. Piscataway, NJ: Transaction Publishers.
Smithson, M. 2015. "Ignorance Studies: Interdisciplinary, Multidisciplinary, and Transdisciplinary". Pp. 385-399 en Routledge International Handbook of Ignorance Studies, editado por M. Gross and L. McGoey. London: Routledge.

Stauffacher, M, N. Muggli, A. Scolobig y C. Moser. 2015. "Framing Deep Geothermal Energy in Mass Media: The Case of Switzerland". Technological Forecasting and Social Change 98: 60-70. http://dx.doi.org/10.1016/j. techfore.2015.05.018

Stengers, I. 2000. The Invention of Modern Science. Minneapolis, MN: University of Minnesota Press.

Stilgoe, J. 2015. Experiment Earth: Responsible Innovation in Geoengineering. London: Routledge.

Tironi, M. 2014. "Atmospheres of Indagation: Disasters and Politics of Excessiveness". Sociological Review 62(S1): 114-134. http://dx.doi.org/10.1111/1467-954X.12126

Tironi, M. 2015. "Disastrous Publics: Counter-Enactments in Participatory Experiments". Science, Technology \& Human Values 40 (4): 564-587. http://dx.doi. org/10.1177/0162243914560649

Van de Poel, I. 2016. "An Ethical Framework for Evaluating Experimental Technology". Science and Engineering Ethics 22 (3): 667-686. http://dx.doi.org/10.1007/s11948-015-9724-3

Vienken, T, S. Schelenz, K Rink y P. Dietrich. 2014. "Sustainable Intensive Thermal Use of the Shallow Subsurface: A Critical View on the Status Quo". Groundwater 53 (3): 356-361. http://dx.doi.org/10.1111/gwat.12206

MATTHIAS GROSS es profesor de Sociología Medioambiental en el Helmholtz Centre for Environmental Research en Leipzig y en la Universidad de Jena, Alemania. Su investigación reciente se enfoca en la evolución de sistemas energéticos alternativos, la centralidad de la ignorancia en la ciencia y en la vida cotidiana, y las prácticas experimentales en la vida social.

MANUEL TIRONI es profesor asociado y coordinador del grupo Estudios Críticos del Antropoceno en el Instituto de Sociología de la P. Universidad Católica de Chile. Es investigador asociado del Centro de Investigación Integrada en Desastres Naturales (CIGIDEN) y del Núcleo Milenio de Investigación en Energía y Sociedad (NUMIES). Su trabajo se ubica en la intersección entre los estudios sociales de la ciencia y tecnología, la sociología medioambiental y la teoría política. Sus últimos proyectos tratan temas de toxicidad, justicia ambiental, conocimiento geológico y ciencia ciudadana. Sus artículos más recientes han sido publicados en Sociological Review, Geoforum y Science Technology \& Human Values, entre otros. Ha sido profesor invitado en las universidades de Goldsmiths - University of London, Copenhague y Warwick. 